\title{
From Design to Experiments of a 2 DOF Vehicle Driving Simulator
}

\author{
Hichem Arioui, Salim Hima, Lamri Nehaoua, René J.V. Bertin, and Stéphane Espié,
}

\begin{abstract}
Driving simulators are more and more used for driver evaluation and/or education. In this paper, we describe the design and the modeling aspects of a 2 DOF low cost motion platform allowing the rendering of the longitudinal and yaw movements. This prototype will be used to study various configurations of motion rendering and the impact of these variants on controllability and on simulator sickness.

The whole motion platform is considered as two coupled systems that are linked mechanically. The first system consists of a motorized rail for the longitudinal movement which is mounted on top of the second system, a motorized turret allowing to rotate the platform.

We present the platform mechanics and a number of experimental studies that have been carried out to obtain a characterization of the platform capabilities and frequency responses as well as to assess platform performance in a classical drive operation. First conclusions and directions of future work are presented.
\end{abstract}

Index Terms-Driving Simulator, Motion Cueing Algorithm, Design and Modeling

\section{INTRODUCTION}

The use of driving simulators is increasingly widespread and adopted by various public and private institutions for conducting research and/or education programs. A driving simulator is a virtual reality tool that allows users to drive in conditions that are safe for the driver as well as for the other road users. Motion cueing platforms were firstly used for aircraft simulators and were democratized for cars and recently for motorcycles [1] [2] [3]. Driving simulators became very accessible through technological progress. Indeed, computers have become more powerful and less expensive. Thus, several simulators of various architectures were built with an aim of either human factors study [4] [5] [6] [7], or to test new car prototypes and functionalities [8] [9] [10] [11], or for driver training and education.

The importance of aircraft and passenger safety, or of reducing the number of prototypes in car design, allows investment in high-cost simulators. This is much less true if the simulators are to be tools for training and/or driver behavioral studies [12] [13] [14]. There is no scientific evidence for the notion that such tools are required to be of high complexity, nor for a required physical fidelity of the motion cueing. Most will agree that inappropriate motion cueing is likely to induce simulator sickness through multisensory conflict. There is however little

Manuscript received January 06, 2010; revised April 15, 2010. This work was supported by the National Agency of Research (ANR) under VIGISIM project.

H. Arioui, S. Hima and L. Nehaoua are with the IBISC-CNRS, Evry, France R.J.V. Bertin and S. Espié are with the INRETS-LCPC, Paris, France scientific evidence on how motion rendering quality impacts the occurrence and development of simulator sickness. For this reason, the INRETS-MSIS (which became INRETS/LCPC LEPSIS) decided to initiate the design of a mobile platform aimed primarily at studying the importance of the modalities of yaw rendering on virtual vehicle control and on simulator sickness.

Dynamic driving simulator systems allow a driver to interact safely with a synthetic urban or highway environment via a motion cueing platform that feeds back the essential inertial components (acceleration and rotation) of the vehicle's movements, in order to immerse the driver partially or completely. The complexity of dynamic driving simulators lies in the fact that the system is composed of interconnected subsystems of different nature (mechatronics, control laws, computer, etc.) of which a human subject is an integral part. Dynamic driving simulators should thus be studied in their entirety, including the human driver. In the present paper, we are interested in the design, mechatronics and identification aspects of the platform, but we do present an initial subjective evaluation of two different configurations by a small number of drivers.

In general, a range of accelerations as large as is experienced during real driving cannot be reproduced on a motion cueing platform. A compromise is to be found between the quality of rendering of various inertial cues and maintaining the platform within its reachable workspace. Therefore, many control strategies were developed [15] [16] [17] [18]. Motion Cueing Algorithms (MCA) were firstly used in flight simulators. Porting them to driving simulators is possible, but terrestrial vehicle dynamics contain much higher frequencies (more abrupt and frequent variations of acceleration) than what is observed in air planes. Besides, driving a vehicle takes place within traffic and unforeseen events (fog, pedestrians, etc.)are more likely to occur, which could create more complex scenarios.

All the considerations cited above will be taken into account in order to facilitate the design of a low-cost motion platform with two degrees of freedom.

In the rest of this paper we present the design, description and modeling aspects of the platform. The identification aspects and the motion control algorithm are explained briefly and followed by a presentation of the experiments that were carried out to characterize motion response frequencies and to obtain driver evaluation from initial, closed loop drive tests. 


\section{Motivation of the Platform Architecture CHOICE}

The choices of simulator structure and motion bases are motivated by the necessity to have a sufficient perception while driving as well as by financial design constraints. Thus, the objective of the simulator project is not to reproduce all of a real vehicle's motions, but only the longitudinal movements, and yaw. This inertial feedback is to be perceived by the human user in the intended applications, which include the study of the effects of yaw cueing on simulator sickness. Indeed, one of the more nauseating maneuvers in a driving simulator is the negotiation of (sharp) curves, especially intersections in complex environments like agglomerations. These maneuvers create important and large-scale changes in the $2 \mathrm{D}$ projection of the virtual world (the so-called fronto-parallel component of the optic flow). On the one hand, repeated exposure to such stimulation may induce disorientation and/or simulator sickness in sensitive individuals. On the other hand, refresh rates in current visual rendering systems are not high enough to minimize angular frame-to-frame displacement of objects, which often leads to rendering or perceptual artifacts that could also induce disorientation and/or simulator sickness. Rotating in front of (or inside) a visual scene devoid of frontoparallel motion may well be different from being stationary with respect to a visual scene that also reproduces angular motion, at least for the second aspect, and in addition to the potential benefits of appropriate yaw cueing in itself. It is known for instance that thresholds for visual detection of course deviations are quite high, often leading to over-correction when a deviation is detected finally, resulting in weaving. This is a controllability issue, but one that is clearly linked to simulator sickness occurrence. A course deviation starts as a heading variation. A rotating base ought to be able to render such variations in a way to deliver cues for which human thresholds and reaction times are low, improving controllability.

In order to reduce the cost of the dynamic simulator, the idea is to eliminate some degrees of motion. In fact, some platforms are proposed with only a longitudinal motion [3], while evaluation tests reveal the insufficiency of this kind of platform. This insufficiency is manifested essentially in simulated movements with a lateral component where incoherence between the visual and inertial perception is observed and leads to driver sickness. This observation has motivated our choice to propose a platform which offers a sufficient inertial perception feedback while still having a low cost of design. In fact, in addition to the longitudinal motion which is important for acceleration and deceleration cues, our platform integrates a mechanism that can generate a yaw motion at full scale.

Moreover, we know that there is a strong correlation between driving simulation sensations and the number of perceptive stimuli [19]. Based on this observation, a reproduction of the dynamic tire-road contact system is implemented in order to assist the driver (actuator mounted on the steering wheel). The modeling part of this last point is not addressed in the present paper. Figure (1) presents the experimented architecture platform which will be described in the next section.

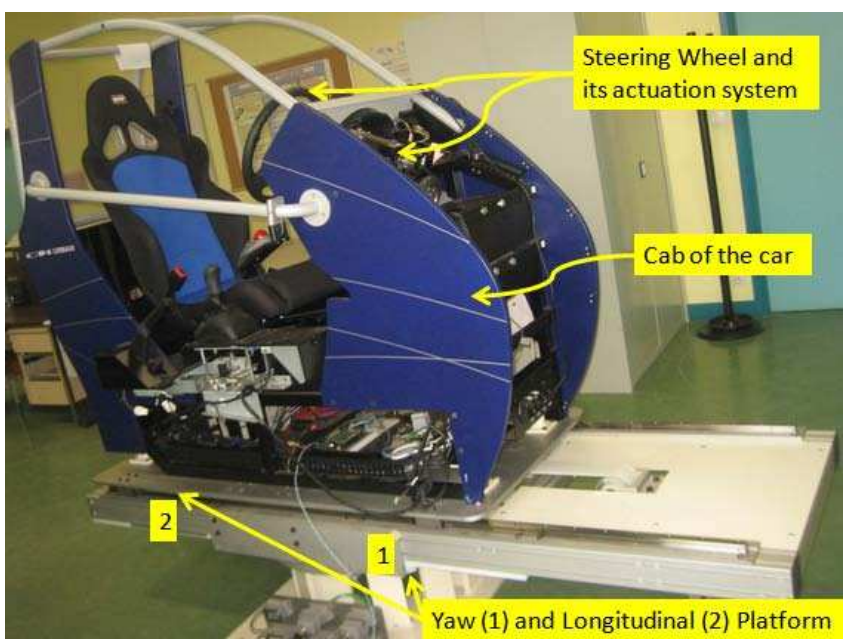

Fig. 1. The experimented 2 DOF simulator platform.

\section{Platform Description}

\section{A. Simulator Architecture}

We present in this paper a mini driving simulator with an acceptable compromise between rendering quality, compactness and cost limitation. The mechatronics components of the proposed solution are described below:

- The cabin consists of an instrumented mobile part moving along a guide way mounted on the platform. It is the interface between the driver and the simulated environment. The cabin is equipped with acceleration and braking pedals, steering wheel, gearbox lever and other classical car control organs (figure 1). These inputs are fed back into the vehicle dynamics model in order to update its several states.

- The acquisition system is composed of an industrial micro controller. This allows the control of actuators in the desired position, speed or torque (used for the steering wheel force feedback). A bidirectional information exchange protocol is defined between this card and the PCs dedicated to vehicle and traffic models. The communication can be performed through either parallel or CAN ports.

- The vehicle model allows the determination of the virtual vehicle states according to the driver's input control. A dynamic model, with a complexity level appropriate for our application and especially the feedback movements, has been developed. It is clear that the dynamic model must at least be able to compute these three variables. We have adopted the well known bicycle model with a more enhanced tire/road contact representation using the Pacejka formula. The vehicle dynamic model concerns the computation of the dynamics and the kinematics as a function of the driver actions (on the accelerator, brake pedal, clutch, etc.) and the road characteristics. In this model, the vehicle is considered as one body with 5DOF (longitudinal, lateral, roll, pitch and yaw). The engine part is modeled by a combined mechanical and behavioral approach [20] based on the vehicle's general characteristics (engine torque curves, clutch pedal position, accelerating proportioning, etc.). After updating the vehicle's state, relevant resulting information is sent to the cabin's dashboard and to the traffic model server. 
The cabin displays (dashboard) and audio systems are not operational at the time of writing.

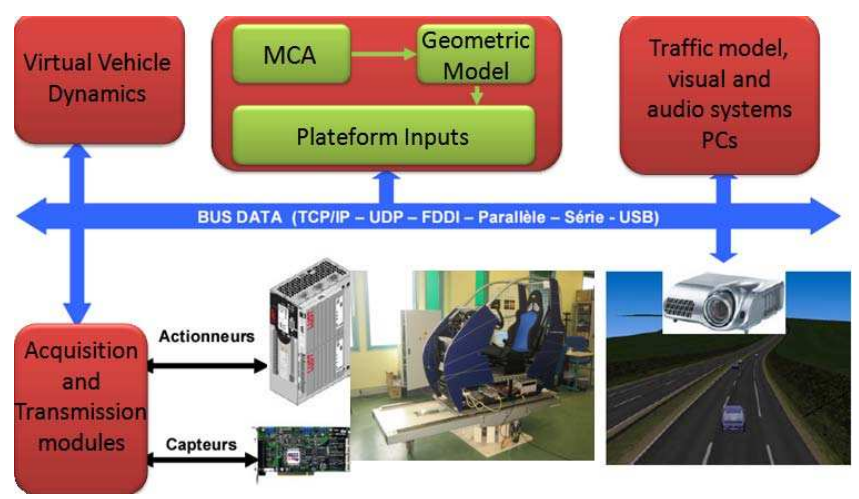

Fig. 2. Simulation Synoptic Architecture.

The platform is equipped with power, sensors and control modules in order to have information feedback on the control system states. The yaw and longitudinal actuators contain servomotors controlled through drives that ensure and provide feedback on angular position and velocity and the output torque. These drives are connected via CAN to a control PC running an appropriate model.

\section{B. Mechanical Description}

The platform is composed of two metallic parts linked mechanically. The upper part consists of the car cabin fixed onto a chassis that can move linearly on the lower part in such a way that the cabin moves either longitudinally (the current configuration) or transversely. The lower part consists of support structure on which is fixed the rotation drive system of the yaw motion (see figures 3 and 4). The overall weight of the upper system (cabin, the sliding plate and an averageweight driver) is approximately $430 \mathrm{~kg}$.

To control yaw and longitudinal motions of the platform, two actuators have been used. Through two sliders, assembled under the two edges of the cabin's base, the platform is able to move on a rail of $1.2 \mathrm{~m}$ length. The movement is generated through a pulley-belt system by a brush less type servomotor SMB 80 with a reduction ratio of 45 . This platform achieves linear accelerations up to $\pm 0.408 \mathrm{~g}$ in steady mode. At peak current, acceleration and speed of $\pm 1.224 \mathrm{~g}$ and $\pm 2.45 \mathrm{~m} / \mathrm{sec}$ respectively are reached.

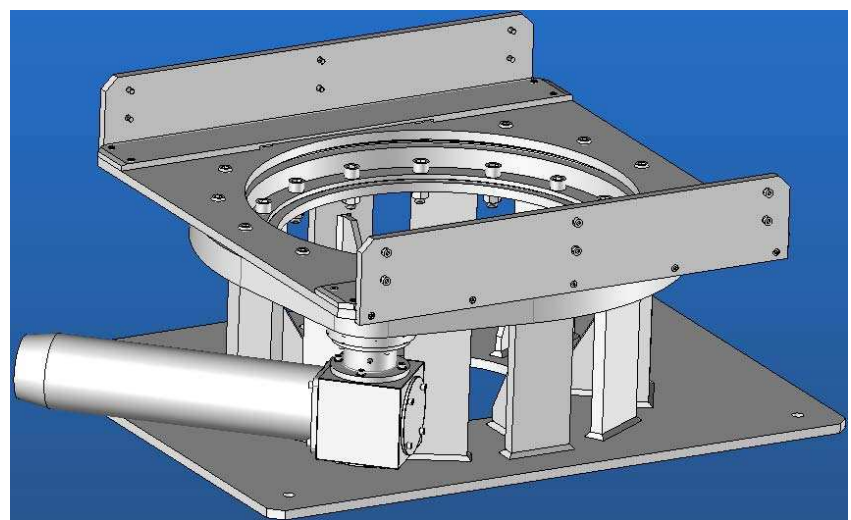

Fig. 3. Upper metallic frame of the longitudinal motion.

The yaw motion is controlled by a rotation system under the vertical structure and driven by a circular ball-screw drive actuated system also operated by an SMB 80 servomotor, this time with a reduction ratio of 139.2 (see figure 4). This system achieves angular accelerations up to $3.971 \% / s^{2}$ in steady mode. At peak current, acceleration and speed of $58.151 \% / \mathrm{s}^{2}$ and $29.075 \%$ respectively are reached.

The axis of yaw rotation of the platform can be changed or adapted to the situation that we want to simulate. That is, the upper part of the platform (and thus the center of the longitudinal motion range) can be moved manually relative to the lower part. This option allows us to study better the impact of the yaw rotation (rotation speed and radius of curvature, etc.) on the perceptional quality of the motion cueing.

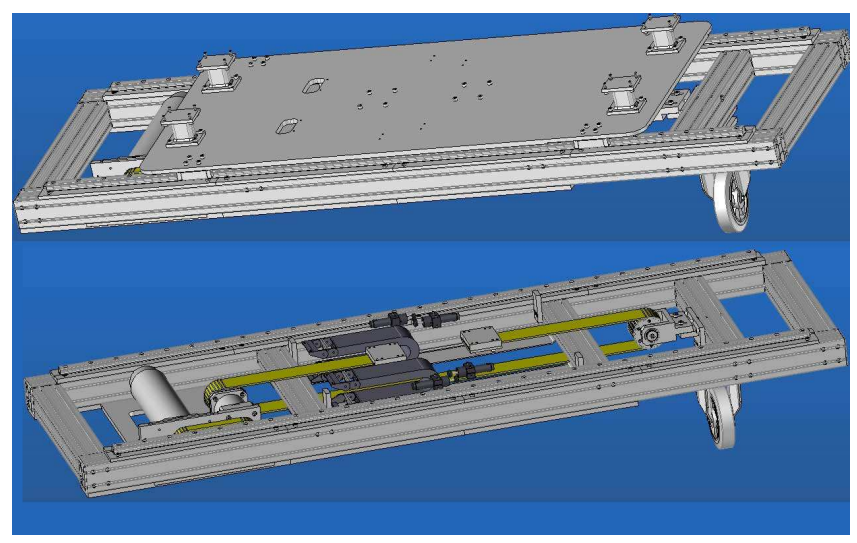

Fig. 4. The circular ball-screw drive actuated system.

\section{Platform Modeling AND IDENTIFICATION}

Control of robotic mechanisms is mostly based on the knowledge of an accurate behavioral model that governs their motions. Indeed, the accuracy of the model depends essentially on the quantification of the phenomena that act on it, and on the precision of its parameters. We devote this section to the derivation of the dynamic model of our platform in response to actuator torques.

\section{A. Platform Kinematic Modeling}

The effect of the front wheel dynamics on that of the whole system is neglected. In fact, we remove the wheel and replace 
it with a resistive torque, resulting from the friction forces of the wheel/ground interaction, and acting on the yaw motion. Hence, the system treated in this paper can be seen as a serial multi body system with three bodies linked by two degrees of freedom, RP manipulator. In this case, three orthonormal frames are used to describe the motion of the platform, see figure 5. Body $B_{0}$ and body $B_{1}$ are linked with a revolute joint parametrized by the variable $q_{1}$. So, the transformation between frame $\mathcal{R}_{0}$ and $\mathcal{R}_{1}$ is given by the rotation matrix:

$$
R=\left(\begin{array}{ccc}
\cos \left(q_{1}\right) & -\sin \left(q_{1}\right) & 0 \\
\sin \left(q_{1}\right) & \cos \left(q_{1}\right) & 0 \\
0 & 0 & 1
\end{array}\right)
$$

Besides, Body $B_{2}$ performs a translation with respect to body $B_{1}$ parametrized by the variable $q_{2}$. Hence, the configuration of the platform can be easily described by the vector $\mathbf{q}=\left(q_{1}, q_{2}\right)$.

Let $\left(x_{G_{1}}, y_{G_{1}}, z_{G_{1}}\right)$ and $\left(x_{G_{2}}, y_{G_{2}}, z_{G_{2}}\right)$ denote, respectively, the positions of the center of mass for bodies $B_{1}$ and $B_{2}$ in their attached frames.

Angular velocities of bodies $B_{1}$ and $B_{2}$ are given by:

$$
\omega_{1}=\omega_{2}=\left(\begin{array}{c}
0 \\
0 \\
\dot{q}_{1}
\end{array}\right)
$$

Then linear velocities of $G_{1}$ and $G_{2}$ are given by:

$$
\begin{aligned}
\mathbf{V}_{G_{1}} & =\omega_{1} \times \mathbf{O}_{1} \mathbf{G}_{1} \\
\mathbf{V}_{G_{2}} & =\mathbf{V}_{O_{2}}+\omega_{2} \times \mathbf{O}_{2} \mathbf{G}_{2}
\end{aligned}
$$

By projecting these expressions in their local frames we find:

$$
\begin{gathered}
\mathbf{V}_{G_{1}}=\left(\begin{array}{c}
-\dot{q}_{1} y_{G_{1}} \\
\dot{q}_{1} x_{G_{1}} \\
0
\end{array}\right) \\
\mathbf{V}_{G_{2}}=\left(\begin{array}{c}
-\dot{q}_{1} y_{G_{2}}+\dot{q}_{2} \\
\left(x_{G_{1}}+q_{2}\right) \dot{q}_{1} \\
0
\end{array}\right)
\end{gathered}
$$

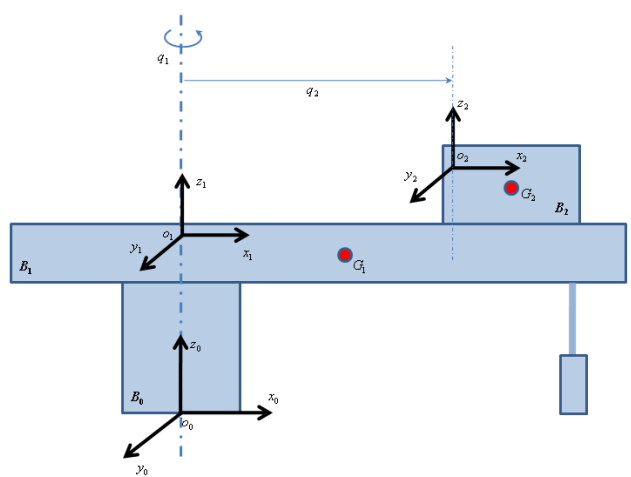

Fig. 5. Frames of the mechanical platform.

\section{B. Platform Dynamics}

Modeling mechanical mechanisms has attracted a great deal of attention for a long time and has attained a great maturity. In fact, these developments have led to very efficient, accurate and rapid algorithms which meet the requirements for robotic applications or computer animation for example and for applications with a large number of degrees of freedom [21].

There exist several methods to derive the dynamics equations of mechanisms such as: Newton-Euler's formalism, Hamilton's formalism, Kane's formalism, etc. In this paper, we have used Lagrange's formalism for its simplicity. The equations of motion can than be obtained using Lagrange's equation for each generalized set of co-ordinates:

$$
\frac{d}{d t}\left(\frac{\partial T}{\partial \dot{q}_{i}}\right)-\frac{\partial T}{\partial q_{i}}=Q_{i}
$$

where $T=V-U$ is the Lagrangian function defined by the difference between the total kinetic energy of the system $V$ and the total potential energy of the system $U$. In the case of the platform presented in this paper, which evolves in the $(X Y)$ plane, $T$ is reduced to only kinetic energy:

$$
T=V=\frac{1}{2} \sum_{i=1}^{2}\left(\mathbf{V}_{G_{i}}^{T} \mathbf{M}_{i} \mathbf{V}_{G_{i}}+\omega_{i}^{T} \mathbf{I}_{i} \omega_{i}\right)
$$

where $\mathbf{M}_{\mathbf{1}}=m_{1}$ eye $(3)$ and $\mathbf{M}_{\mathbf{2}}=m_{2}$ eye $(3)$ are respectively, body $B_{1}$ and $B_{2}$ matrix of masses, $\mathbf{I}_{\mathbf{1}}=$ $\operatorname{diag}\left(I_{1_{x x}}, I_{1_{y y}}, I_{1_{z z}}\right)$ and $\mathbf{I}_{\mathbf{2}}=\operatorname{diag}\left(I_{2_{x x}}, I_{2_{y y}}, I_{2_{z z}}\right)$ are respectively, the moment of inertia tensors of bodies $B_{1}$ and $B_{2}$ expressed in their local frames.

By replacing equations (4) and (5) in equation (7), the previous expression becomes:

$$
\begin{aligned}
T= & \frac{1}{2}\left(m_{1}\left(x_{G_{1}}^{2}+y_{G_{1}}^{2}\right)+I_{1_{z z}}+m_{2} y_{G_{2}}^{2}+m_{2}\left(x_{G_{1}}+q_{2}\right)^{2}\right. \\
& \left.+I_{2_{z z}}\right) \dot{q}_{1}^{2}+\frac{1}{2} m_{2} \dot{q}_{2}^{2}-m_{2} y_{G_{2}} \dot{q}_{1} \dot{q}_{2}
\end{aligned}
$$

It is straightforward to show that, by application of Lagrange's formalism, equation (6), the platform equations of motion can take the following from:

$$
\mathbf{M}(\mathbf{q}) \ddot{\mathbf{q}}+\mathbf{C}(\mathbf{q}, \dot{\mathbf{q}})=\mathbf{Q}
$$

where $\mathbf{M}(\mathbf{q})$ is the system inertia matrix given by:

$$
\mathbf{M}(\mathbf{q})=\left(\begin{array}{ll}
m_{11} & m_{12} \\
m_{21} & m_{22}
\end{array}\right)
$$

such as:

$$
\begin{aligned}
m_{11}= & m_{1}\left(x_{G_{1}}^{2}+y_{G_{1}}^{2}\right)+I_{1_{z z}} \\
& +m_{2} y_{G_{2}}^{2}+m_{2}\left(x_{G_{2}}+q_{2}\right)^{2}+I_{2 z z} \\
m_{12}= & m_{21}=-m_{2} y_{G_{2}} \\
m_{22}= & m_{2}
\end{aligned}
$$

and $\mathbf{C}(\mathbf{q}, \dot{\mathbf{q}})$ is a vector of centrifugal and Coriolis forces:

$$
\mathbf{C}(\mathbf{q}, \dot{\mathbf{q}})=\left(\begin{array}{c}
2 m_{2}\left(x_{G_{2}}+q_{2}\right) \dot{q}_{1} \dot{q}_{2} \\
-m_{2}\left(x_{G_{2}}+q_{2}\right) \dot{q}_{1}^{2}
\end{array}\right)
$$


$\mathbf{Q}$ is the external forces/torques vector acting on the platform including traction and friction forces/torques:

$$
\mathbf{Q}=\left(\begin{array}{c}
\tau_{1}-\tau_{f_{1}} \\
\tau_{2}-\tau_{f_{2}}
\end{array}\right)
$$

$\tau_{1}$ and $\tau_{2}$ are obtained by multiplying the effective torque, delivered by the motors, by the reduction ratio of the gear systems integrated into their respective joints:

$$
\begin{aligned}
& \tau_{1}=K_{1} \tau_{a_{1}} \\
& \tau_{2}=K_{2} \tau_{a_{2}}
\end{aligned}
$$

where: $K_{1}$ and $K_{2}$ are about 139.2 and 45 respectively. $\tau_{f_{1}}$ and $\tau_{f_{2}}$ are the friction torques. These torques are modeled as a combination of dry and viscous frictions:

$$
\tau_{f_{i}}=K_{s_{i}} \operatorname{sign}\left(\dot{q}_{i}\right)+K_{v_{i}} \dot{q}_{i} \quad i=1,2
$$

In the next section, we will discuss the identification of all the parameters of the developed dynamic model, and the used approach. The frequency characterization of the system dynamics is also done to obtain an idea of the system 's transition capabilities.

\section{Identification Process}

We expose here the identification process used to estimate frictions (dry and viscous), mass and inertia parameters. First, we consider the estimation of dry and viscous frictions $K_{s_{i}}$ and $K_{v_{i}}$ for the two DOF. To this extent, a simple method is used which consists of driving the simulator platform with step and ramp position profiles. For a step position profile, speed and acceleration terms are canceled. Consequently, the $K_{s_{i}}$ parameters can be identified and equation (15) becomes:

$$
\tau_{f_{i}}=K_{s_{i}} \operatorname{sign}\left(\dot{q}_{i}\right) \quad i=1,2
$$

In the same manner, for a ramp position profile, acceleration terms are canceled, the $K_{v_{i}}$ parameters can be identified, and:

$$
\tau_{f_{i}}-K_{s_{i}} \operatorname{sign}\left(\dot{q}_{i}\right)=K_{v_{i}} \dot{q}_{i} \quad i=1,2
$$

Once the friction forces are determined, inertial parameters are identified by driving the simulator platform with a chirp sinus position trajectory. To do this, the dynamic model can be written in linear form with respect to the different parameters to be estimated, as follows:

$$
\begin{aligned}
& \theta_{1}=\left(m_{1}+m_{2}\right)\left(x_{G_{1}}^{2}+y_{G_{1}}^{2}\right)+I_{1_{z z}}+I_{2_{z z}} \\
& \theta_{2}=2 m_{2} x_{G_{2}} \\
& \theta_{3}=m_{2} \\
& \theta_{4}=-m_{2} y_{G_{2}}
\end{aligned}
$$

and

$$
\begin{array}{r}
\mathbf{M}(\mathbf{q}) \ddot{\mathbf{q}}=\mathbf{C}_{1}(\mathbf{q}, \dot{\mathbf{q}}, \ddot{\mathbf{q}}) \boldsymbol{\Theta} \\
\mathbf{C}(\mathbf{q}, \dot{\mathbf{q}}) \dot{\mathbf{q}}=\mathbf{C}_{2}(\mathbf{q}, \dot{\mathbf{q}}, \ddot{\mathbf{q}}) \boldsymbol{\Theta} \\
\mathbf{Q}=\left(\begin{array}{c}
\tau_{1}-\tau_{f_{1}} \\
\tau_{2}-\tau_{f_{2}}
\end{array}\right)
\end{array}
$$

where:

$$
\begin{aligned}
\mathbf{C}_{1} & =\left(\begin{array}{cccc}
\ddot{q}_{1} & q_{2} \ddot{q}_{1} & q_{2}^{2} \ddot{q}_{1} & \ddot{q}_{2} \\
0 & 0 & \ddot{q}_{2} & \ddot{q}_{1}
\end{array}\right) \\
\mathbf{C}_{2} & =\left(\begin{array}{cccc}
0 & \dot{q}_{1} \dot{q}_{2} & 2 q_{2} \dot{q}_{1} \dot{q}_{2} & 0 \\
0 & -\frac{1}{2} \dot{q}_{1}^{2} & -q_{2} \dot{q}_{1}^{2} & 0
\end{array}\right)
\end{aligned}
$$

The experimental tests have led, after light calculation, to the parameters summarized in the following table:

\begin{tabular}{|c|c|c|c|}
\hline Mass $m_{1}$ & Mass $m_{2}$ & Inertia $I_{1_{z z}}+I_{2 z z}$ & $x_{G_{1}}^{2}+y_{G_{1}}^{2}$ \\
\hline $415.98 \mathrm{~kg}$ & $359.32 \mathrm{~kg}$ & $4.69 \mathrm{~kg} . \mathrm{m}^{2}$ & $0.162 \mathrm{~m}^{2}$ \\
\hline \hline$x_{G_{2}}$ & $y_{G_{2}}$ & Viscous $K_{v_{1}}$ & Viscous C. $K_{v_{2}}$ \\
\hline $79.76 \mathrm{~cm}$ & $-1.07 \mathrm{~cm}$ & $0.0438 \mathrm{~N} . \mathrm{s} / \mathrm{m}$ & $0.175 \mathrm{~N} . \mathrm{s} / \mathrm{m}$ \\
\hline \hline Dry F. $K_{s_{1}}$ & $0.2361 \mathrm{~N}$ & Dry F. $K_{s_{2}}$ & $0.6909 \mathrm{~N}$ \\
\hline
\end{tabular}

The given parameters are the platform's inertial and geometric parameters which are constant in all platform working ranges, except for the co-ordinates of the gravity center. These have been identified in a particular configuration (zero yaw angle and mobile platform attached to a known distance from local frame) and with a "standard" driver (1m75 and $70 \mathrm{~kg}$ ). Therefore, the inertial parameters change with the motion platform (these last are updated by the dynamic equation).

\section{Motion Cueing Algorithm}

The physical limits of the platform's workspace do not allow to reproduce the full scale of the actual movements as calculated by the virtual vehicle model. Therefore, we must generate platform trajectories which remain inside the reachable workspace while satisfying a driving behavior that is as close as possible to what can be observed in real situations. These trajectories (inputs of the motion algorithm unit) are generated from a numerical vehicle dynamic model. Before feeding the platform by these reference signals, they undergo a transformation in order to adapt them to platform physical constraints. This transformation consists of filtering the longitudinal acceleration and yaw rate to get the high frequencies (short displacements) which represent the useful information to be cued to the driver. In addition, the platform should regain the initial position in a smooth way and below the driver's perceptual threshold. In this way we prepare the platform for the next motion. This is the role of the motion cueing algorithms.

On a mobile driving simulator, real vehicle motion is reproduced in part by means of a moving base platform that stimulates the human kinesthetic receptors and thus provides drivers with appropriate cues. However, the restricted mechanical space does not allow one-to-one restitution of real vehicle motion. The platform of a simulator must travel considerable distances to reproduce sustained lateral or longitudinal accelerations. Poor simulation of these accelerations, especially for emergency braking, is one of the most important limitations of simulators. Consequently, longitudinal and lateral accelerations are firstly scaled with a scale factor inferior to one. This scale factor is chosen in a manner to maintain an acceptable level of realism. Next, the motion cueing algorithms are used to reproduce the transitory components of the linear 
accelerations, which are sufficient to provide a minimum of appropriate stimulation to the simulator driver.

More precisely, motion cueing algorithms are based on the concept of frequency separation of the linear accelerations and the angular velocities [3] (figure 6). In the typical case of a 6 DOF Stewart platform, the transient components (HF) are reproduced directly by linear and angular motion of the platform. The sustained components $(\mathrm{BF})$ are rendered by the tilt coordination technique. Constant linear and angular velocities are rendered directly by visual projection. In this case, the mobile platform is returned back to its neutral position which allows to have enough workspace to simulate new accelerations. This return, known as the Washout, must respect the acceleration perception thresholds to avoid a false cueing motion [22] [23] [24]. The platform discussed here lacks the DOF needed to simulate sustained components through tilt co-ordination (but might not need this technique for yaw rotation due to its working range).

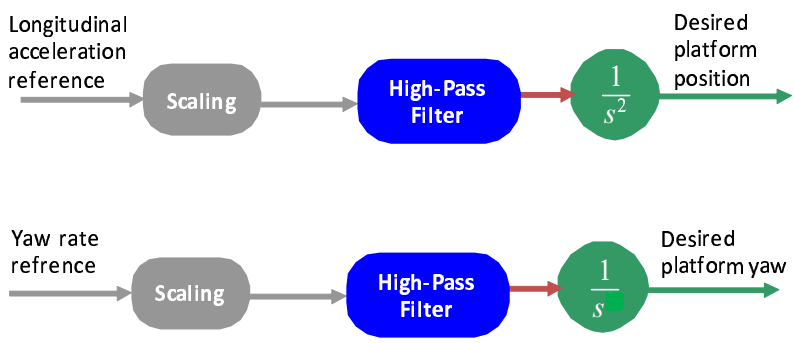

Fig. 6. Motion cueing algorithm for the present platform

Frequency separation is achieved by using linear filters. For the present simulator, we aim to actuate the longitudinal movement and the yaw rotation. Hence, we use one high-pass filter for each of the 2 DOF. Next, the filtered acceleration and yaw rate are integrated twice and once respectively to obtain the longitudinal position $X_{p}$ and yaw rotation $\psi_{p}$ of the simulator platform as following:

$$
\begin{gathered}
\frac{X_{p}}{\ddot{X}_{v}}=\frac{1}{s^{2}} \frac{s^{3}}{\left(s^{2}+2 \zeta_{x} \omega_{x, 1} s+\omega_{x, 1}^{2}\right)\left(s+\omega_{x, 2}\right)} \\
\frac{\psi_{p}}{\dot{\psi}_{v}}=\frac{1}{s} \frac{s^{2}}{s^{2}+2 \zeta_{\psi} \omega_{\psi} s+\omega_{\psi}^{2}}
\end{gathered}
$$

where, $\ddot{X}_{v}, \dot{\psi}_{v}$ are the longitudinal acceleration and yaw rate as calculated by the virtual vehicle dynamics. $\zeta_{x}, \omega_{x, 1}$, $\omega_{x, 2}, \zeta_{\psi}$ and $\omega_{\psi}$ are filter parameters. First, $\zeta_{x}$ and $\zeta_{\psi}$ are chosen to give an over-damped washout response in order to avoid false cues. Next, for a given $\zeta$, the variables $\omega_{x, 1}$ and $\omega_{\psi}$ are determined depending on the platform's mechanical and driver's perceptual constraints. Identical to the approach proposed in [13], $\omega_{x, 1}$ and $\omega_{\psi}$ must satisfy the following constraints:

$$
\begin{aligned}
& \omega_{i} \xi_{i}<\Sigma_{i_{\max }} \\
& \omega_{i}^{2} \xi_{i}^{2}<v_{s} \\
& \omega_{i}^{3} \xi_{i}^{3}<a_{s} \\
& 2 \zeta_{i} \omega_{i}<f_{0}
\end{aligned}
$$

where, $\xi_{i}=\exp \left[\frac{\zeta_{i}}{\sqrt{\zeta_{i}^{2}-1}} \ln \left(\zeta_{i}-\sqrt{\zeta_{i}^{2}-1}\right)\right] . \Sigma_{i_{\text {max }}}$ is the maximum allowed platform displacement and rotation. $v_{s}$ and $a_{s}$ are respectively the velocity and the acceleration thresholds of the vestibular system [25]. $f_{0}$ is the total friction. $\omega_{i}$ denotes $\omega_{x, 1}$ or $\omega_{\psi} \cdot \zeta_{i}$ denotes $\zeta_{x}$ or $\zeta_{\psi}$. Finally, $\omega_{x, 2}$ is tuned by trialerror to take into account drivers subjective evaluations. Based on the developed approach, the used parameters are:

\begin{tabular}{|c|c|c|c|}
\hline Longitudinal acceleration & $\zeta_{x}=2$ & $\omega_{x, 1}=0.5$ & $\omega_{x, 2}=10$ \\
\hline Yaw rate & $\zeta_{\psi}=2$ & $\omega_{\psi}=0.5$ & \\
\hline
\end{tabular}

\section{EXPERIMENTAL RESULTS}

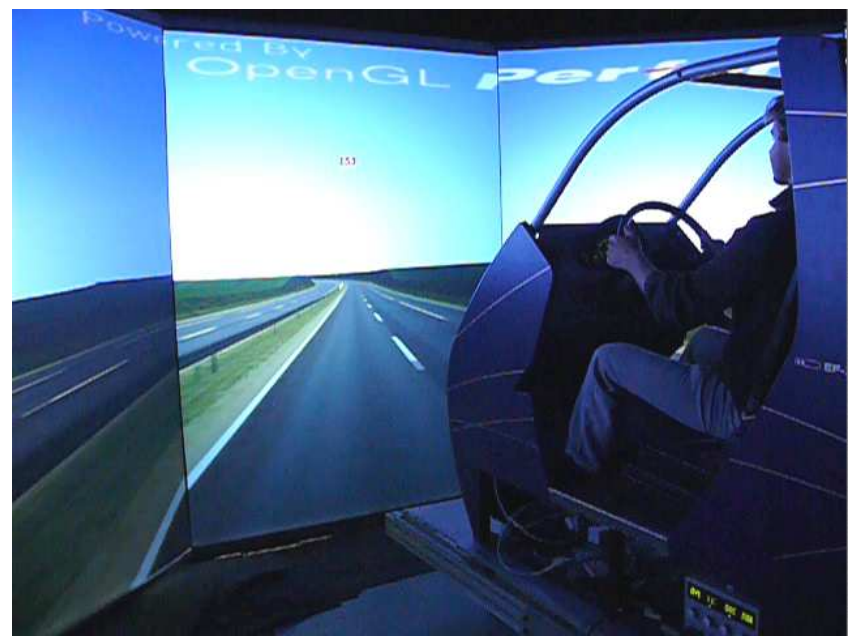

Fig. 7. Experimented driving simulator and its virtual environment

\section{A. Complete Tests with Driver}

In the present section, we show the results of tests made on the developed simulator (figure 7) with a driver. The goal here is to show the actuation system's ability to achieve desired maneuvers in the imposed simulator workspace. Firstly, a moving base simulator has been implemented with the moving base rendering longitudinal position and yaw rotation. In this case, we used the classical algorithm for the longitudinal component given by equations 22 and 23 . 


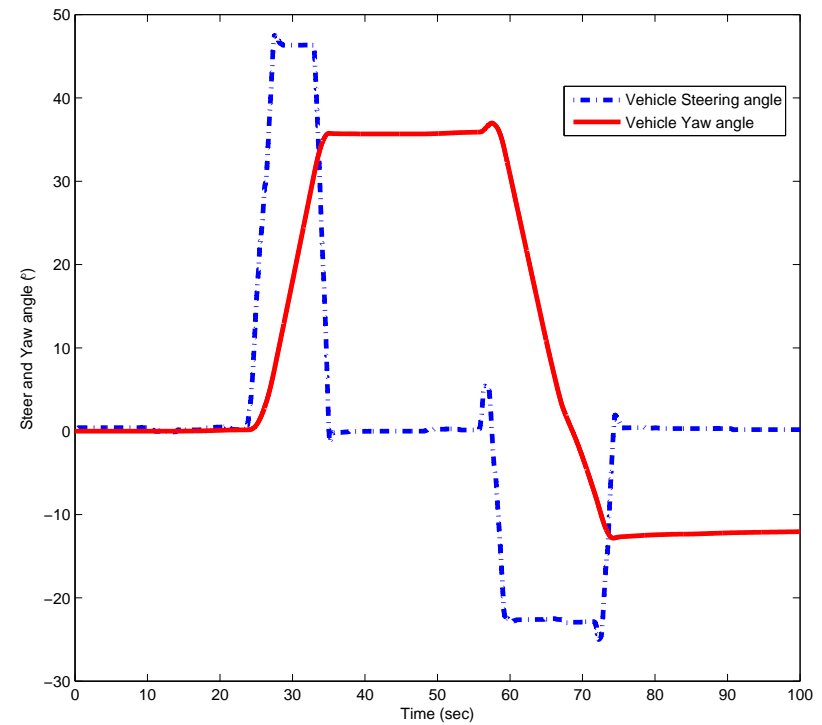

Fig. 8. Steering wheel and Yaw angles of the virtual vehicle

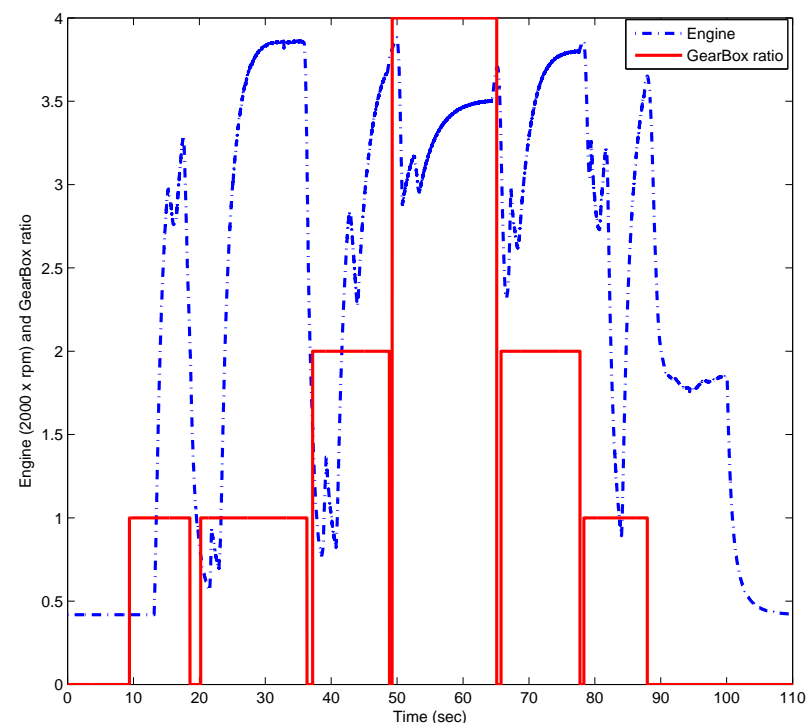

Fig. 9. Engine (x $2000 \mathrm{rpm}$ ) and gearbox ratio during drive

In this experiment, the various actions of the driver are used to calculate the longitudinal acceleration and speed of the virtual vehicle (Figures 8 and 9). More precisely, the scenario consists of a set of accelerations, decelerations and braking maneuvers. The computed longitudinal acceleration and angular yaw velocity through the vehicle dynamic model are used as reference inputs for the classical washout algorithm (MCA). Figure 8 sketches respectively the steering wheel position and the yaw angle calculated by the vehicle model, while Figure 9 represents gearbox position controlled directly by the driver and the the resulted engine to these maneuvers.

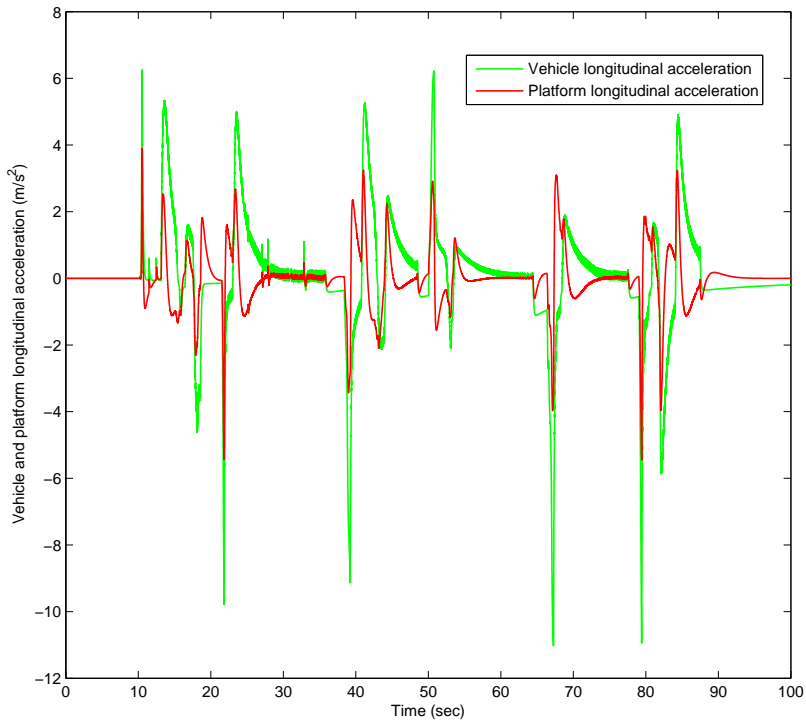

Fig. 10. Vehicle and platform longitudinal acceleration during drive

Figure 10 highlights quantitative similitudes and differences between the vehicle longitudinal acceleration (delivered before the MCA bloc) and restituted one. The used classical algorithm is well suitable for the restitution of the acceleration on-set phases, except that some false cues generated by the linear propriety of the high-pass filters are still existing specially during braking maneuvers.

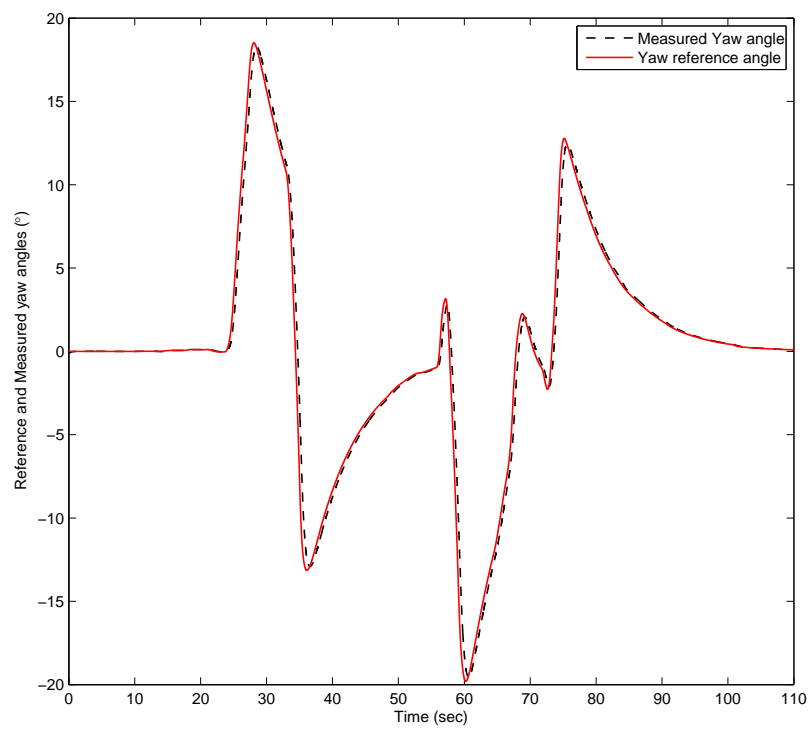

Fig. 11. Reference and Measured yaw angles of the platform motion

Figures 11 and 12 illustrate respectively the reference and the measured yaw angle and longitudinal displacements of the platform. We can see for these two motions the effect of the washout filter that translates acceleration's high frequencies to the platform and brings the platform translation position and 
yaw angle to their origins, with very slow motions, after each acceleration/deceleration phases.

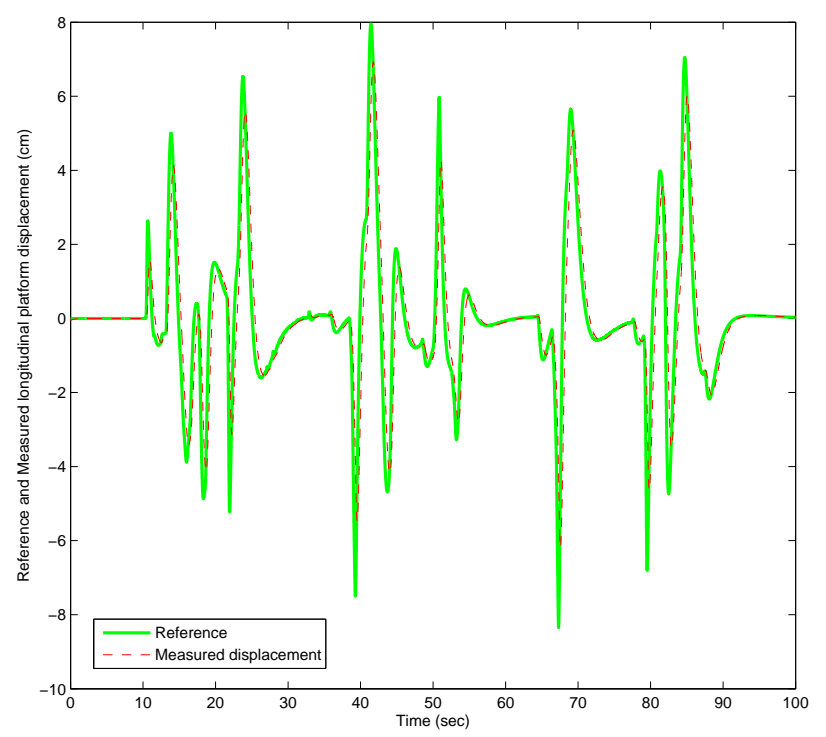

Fig. 12. Reference and Measured longitudinal displacement of the platform's cabin

We find that the motion platform is able to reproduce all (or almost) transitory motions imposed by the driver. It should be noted that this dynamic is quite fast. The simulator does have a minor weakness in the reproduction of high acceleration and longitudinal displacement, however, but the movements felt by the drivers remain acceptable. These remarks constitute a global evaluation of the whole system and allow us to conclude that the actuator's performance is appropriate its intended application.

\section{B. Study of Yaw Motion Impact}

Next, a moving base simulator has been implemented with the moving base rendering yaw only. This implementation allows to evaluate different ways of rendering the angular component of driving a virtual car. For this, a coupling was made with the INRETS simulator software architecture, ArchiSim2. This software is a tool, developed at INRETS, for the simulation of road traffic. In Archisim, traffic phenomena come from individual actions and interactions of the various actors of the road situation. Archisim is a behavioral simulation model implemented following multi-agent principles: simulated drivers in virtual vehicles and pedestrians are agents. For our driving simulator, Archisim is the traffic generator that allows the projection of an interactive visual driving environment. For further information see [26].

In the current implementation, the position and orientation of the virtual car (given by the dynamic model) are calculated and sent to ArchiSim2 for updating the visual scene. The scene is projected onto 5 screens ( $1 m 87$ x $2 m 55)$ positioned around the yaw platform, with the central screen in front of the platform's $0^{\circ}$ heading. In case of a static simulator, the heading sent to ArchiSim2 is the calculated vehicle heading in the virtual world, and this heading is used to set the orientation of the virtual observer used to calculate the $2 \mathrm{D}$ projection. Thus, when the driver negotiates a curve, s/he remains stationary in the real world, and the projected virtual world turns around him. For the current moving base implementation, we subtract the platform's orientation (rotation in the physical world) from the vehicle heading. The $0^{\circ}$ heading in the real and in the virtual worlds coincide with and are mapped to the meridian of the central screen. This approach allows to maintain coherence between platform orientation and the projection of the virtual world. If the driver were to perform a pure rotation in place with 1:1 yaw rendering (Mode 2 below), the platform would turn and the image on the screens would remain perfectly stationary.

In a first evaluation of this system, three subjects have driven the simulator on a $(2 \times 2)$ lanes highway with straight sections and some sweeping curves, in absence of any traffic. They were instructed to drive at a steady speed, keeping to their lane. There were 3 different rendering modes determining the platform rotation angle in time:

- Mode 1: rendering of yaw speed (the 1st temporal derivative of the virtual car's yaw);

- Mode 2: rendering of the virtual car yaw (heading) on a 1:1 scale;

- Mode 3: no rendering, platform immobilized.

Mode 2 was possible due to the fact that the mean heading change in the virtual environment we used is sufficiently close to $0 \circ$. This mode was chosen for the platform's initial evaluations as it is of particular interest to us, not only because our moving base supports it in contrast to a typical hexapod moving base. Indeed, one of the ideas behind the novel and unusual design is the notion that turning inside a stationary visual is likely not the same as being stationary while the surrounding visual rotates. The visual stimulation may be (almost) identical, but the input from the other senses may well be different enough to influence simulator sickness incidence rates - hopefully in beneficial ways. Note that the two dynamic modes do not correspond to commonly used motion control algorithms that are required on hexapod moving bases. In Mode 2, no washout algorithm is required to realign the platform with the $0^{\circ}$ heading. In fact, realignment would be problematic. While sub-threshold could easily be triggered if (average) heading remains constant for a given duration, how to handle a subsequent heading change, especially during the realignment rotation? Mode 1 is the simplest adaptation of Mode 2 to a rendering strategy that would support a realigning washout filter more easily.

The subjects were all able to adapt to driving in all of the 3 modes. Their testimonies, in summary:

- Mode 1: the movement can be sensed easily, probably too much so: the effect is too important. It seems that one has little influence over its amplitude or that it does not depend to a large extent on the steering wheel angle (" on-off" effect). One subject also noticed a feeling of driving side ("crab") wise, as if on ice, probably due to 
a rendering delay. The same subject also remarked on the fact that references fixed in the real world (including the between-screen joints) allow to detect platform movements even if they are otherwise undetectable, reducing the illusion,

- Mode 2: all subjects judged this mode to be easier, more coherent, without the "on/off" effect, and allowing finer movements, and thus a better controllability of the trajectory. The illusion would have to be improved at low speed, however,

- Mode 3: comparable to mode 2, but after having driven with yaw rendering, its absence was felt as a lack, making mode 2 the preferred mode in this evaluation.

While the data from this first evaluation does not lend itself to much quantitative analysis, we thought it interesting to show some of the recorded data, and to point out a few possible links between it, and the subjective assessments given above.

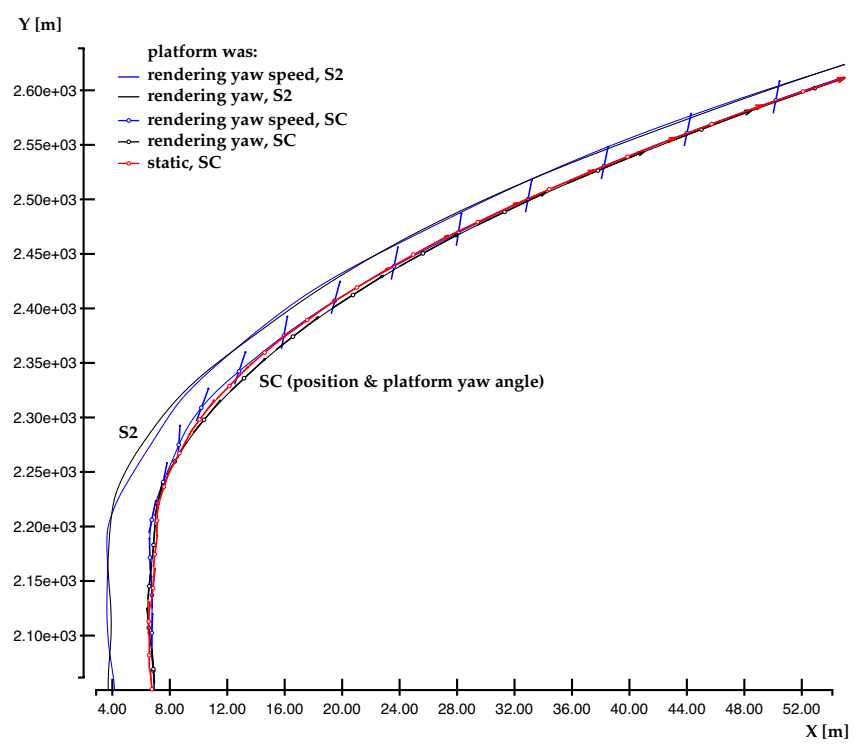

Fig. 13. Entry and exit of the first curve, a few hundred meters after starting to drive. The SC curves have arrows representing the platform orientation in space at the indicated position (and the virtual heading for Mode 3, in red).

Figures 13 and 14 show the top view of the trajectories taken by two subjects (S2 and SC) with different modes of yaw rendering. In blue, the platform rendered the derivative of the virtual vehicle's yaw orientation, in black, yaw was rendered directly and in red, the platform was immobilized.

It can be seen that both subjects have a rather consistent driving strategy, they took trajectories that are very close and similar. However, effects appear to be visible of the negative effects of Mode 1 motion cueing, mentioned above. The reported lack of influence over amplitude (lack of control) may explain the apparent variability in heading (blue SC arrows) and trajectory irregularities (both blue curves) compared to trajectories made in the other two modes (black and red curves). The control difficulties may indeed diminish with time as can be expected, and rather quickly, as the observable variabilities seem to be less important in figure 14 than in figure 13.

Figures 15 show the evolution of four dynamic driving and rendering parameters as a function of the position along the $\mathrm{Y}$ axis in the virtual environment ("depth"). The interval shown corresponds to the section between the entry into the first and the exit out of the second curve. The data shown is from subject SC, colors as in figure 13. The controllability issue when the platform renders yaw rate, a paradigm judged to be more difficult (Mode 1) may explain the fact that the (blue) platform angle curve isn't exactly close to the expected shape (panel 4). Curiously, VR yaw angle variability does not appear to be higher than it is in Mode 2 (blue and black curves, panel 3). Variability in the steering wheel angle may actually be slightly lower in Mode 1 than in Mode 2, which might indicate a more careful control strategy.

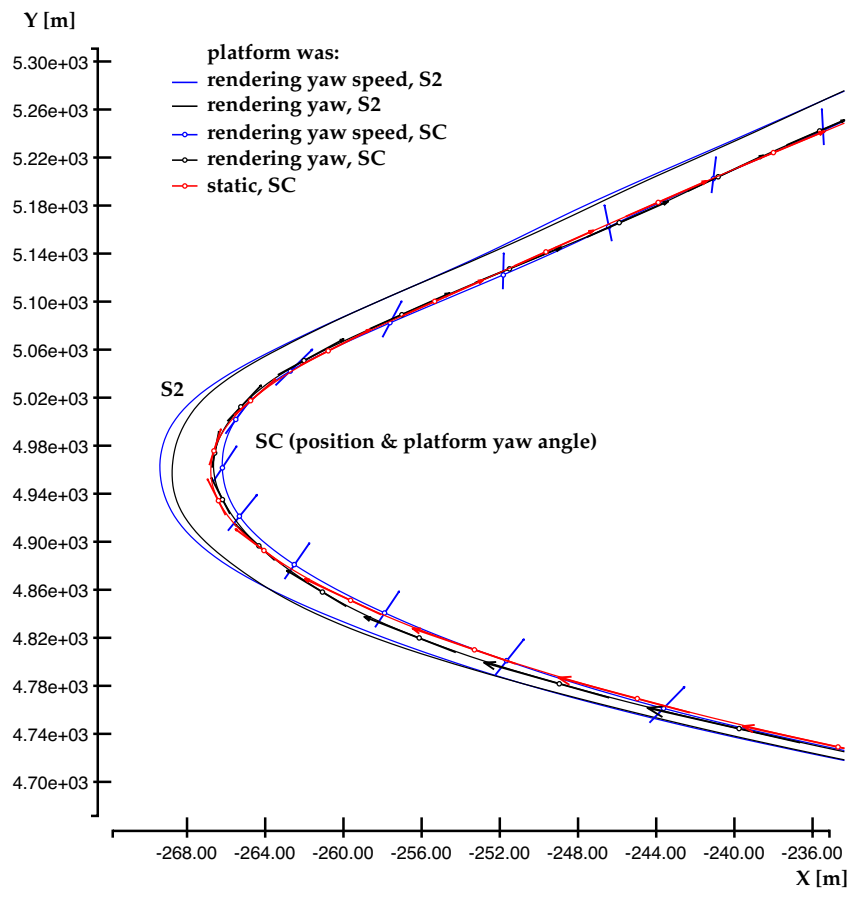

Fig. 14. Entry and exit of the second curve, several kilometers into the drive 

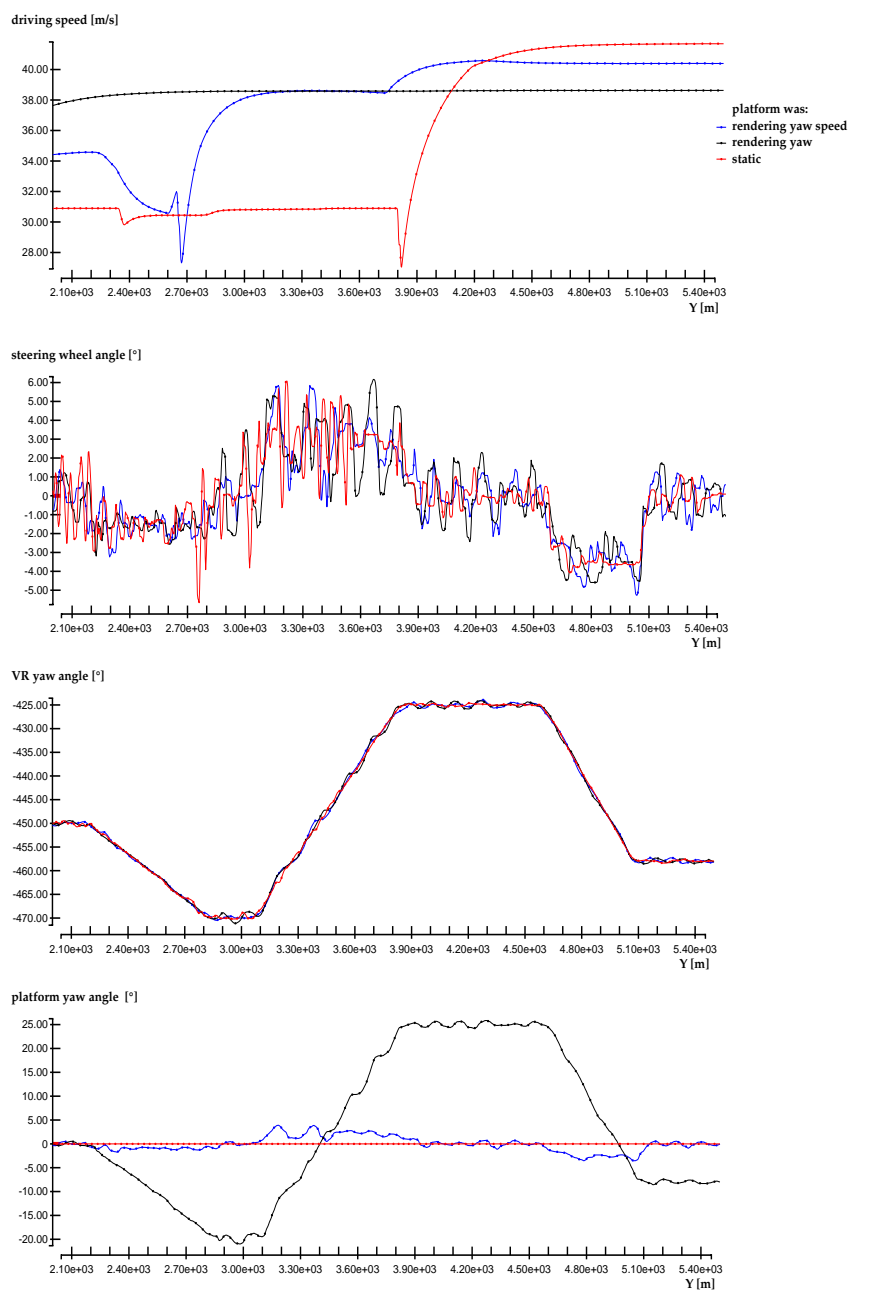

Fig. 15. Time series of driving speed, steering wheel angle, virtual yaw angle (car heading) and platform angle over the periods shown in figures 13 and 14 and in-between, shown as a function of displacement along the Y-axis (depth). Data shown is from subject SC; Mode 1 in blue (platform angle = VR yaw rate), Mode 2 in black (platform angle = VR yaw angle), Mode 3 in red (platform stationary in straight ahead position).

\section{CONCLUSions AND Future WORK}

We proposed a 2 DOF low cost platform for a dynamic driving simulator the design of which is based on perceptual considerations related to driving a vehicle. Although the devices allows only partial restitution of dynamic inertial effects, pertinent cues have been taken into account, namely longitudinal and yaw motions, as well as a steering wheel force feedback (not discussed in this paper). We showed that the platform allows for acceptable driving realism and to observe acceptable driving behavior.

The designed platform has two degrees of freedom. The first one makes it possible to drive the simulator cabin in a front/rear translation. The second allows to produce yaw rotations. The combination of the two movements (translation and yaw rotation) may give the illusion of acceleration variation:

- Static configuration (no motion) with visual rendering alone.

- Longitudinal or lateral (the cabin can be mounted in this orientation on the sliding base).

- Yaw motion alone.
- Combination between longitudinal or lateral with yaw motion.

The previous configurations are enough to conduct many evaluation tests, with subjective and objective measurements.

In order to improve trajectory tracking, a dynamic model of the platform is developed and inertial parameters are identified. The experiments made in closed loop mode are very satisfactory in terms of longitudinal accelerations and yaw rates.

We advocate a low-cost approach to driving simulator design, which we justifies as follows. Existing car driving simulators, with similar (or better) mobility than our platform, generally use Gough-Stewart parallel platforms which are excessively expensive. Comparison of perception quality between these structures is to be studied regarding to psychophysical tests.

Multiple experimentations were completed satisfactorily and allowed us to reach our first objectives. For a complete validation of a simulator using the dynamic platform, psychophysical studies are necessary in order to answer the remaining questions on such issues as driver comfort, simulator controllability, etc.

First conclusions on the impact of the yaw rendering on the driving experience suggests that yaw should be rendered on our platform based on (1:1) vehicle heading rather than on (1:1) yaw rate. This would require to set up a novel washout filter to recenter the platform while driving. A properly conducted study will have to quantify the effect of different rendering approaches on simulator controllability, subject preference and through that the effect on simulator sickness.

This study will build on an earlier study of visuo-vestibular interactions in the perception of yaw rotation. One of the results of this study suggests that subjects disregard scale to a large extent when car heading is being rendered, i.e. one might be able use rotations much smaller than 1:1 rendering would require, possibly any above-threshold rotation in the appropriate direction. If this result is confirmed in the context of our driving simulator, the rendering algorithm could be quite simple indeed.

\section{REFERENCES}

[1] Y. Miyamaru, G. Yamasaki, and K. Aoki, "Development of a motorcycle riding simulator," Society of Automotive Engineers of Japan, vol. 23, pp. 121-126, 2002.

[2] D. Ferrazzin, F. Barnagli, C. Avizzano, G. Pietro, and M. Bergamasco, "Designing new commercial motorcycles through a highly reconfigurable virtual reality-based simulator," Journal of Advanced Robotics, vol. 17 , no. 4, pp. 293-318, 2003.

[3] L. Nehaoua, H. Arioui, H. Mohellebi, and S. Espié, "Motion Cueing Algorithms for Small Driving Simulator," in Proceedings 2006 IEEE International Conference On Robotics and Automation (ICRA06), Orlando, Florida, May 2006, pp. 3189-3194.

[4] P. Grant, B. Artz, J. Greenberg, and L. Cathey, "Motion Characteristics of the VirTtex Motion System," in Proceedings of the 1st HumanCentered Transportation Simulation Conference, IOWA, USA, 2001.

[5] L. D. Chen, Y. Papelis, G. Waston, and D. Solis, "NADS at the University of IowA: A tool for driving safety research," in Proceedings of the 1st Human-Centered Transportation Simulation Conference, IOWA, USA, 2001.

[6] G. Reymond and A. Kemeny, "Motion cueing in the RENAult driving simulator," in Vehicule System Dynamic, vol. 34, Paris, France, Oct. 2000, pp. 249-259. 
[7] L. Sung, K. Ha, C. H. Cho, and L. J. Lee, "The Kookmin University Driving Simulators for Vehicle Control System Development and Human Factor Study," in Driving Simulation Conference (DSC04), Paris, France, 2004.

[8] G. Reymond, A. Heidet, M. Canry, and A. Kemeny, "Validation of RENAULT's Dynamic Simulator for Adaptive Cruise Control Experiments," in Proceedings of the Driving Simulator Conference (DSCO0), Paris, France, 2000, pp. 181-191.

[9] M. Dagdelen, G. Reymond, A. Kemeny, and M. Bordier, "MPC based motion cueing algorithm: development and application to the ULTIMATE driving simulator," in Proceedings of the Driving Simulator Conference (DSC04), Paris, France, 2004.

[10] D. Stall and S. Bourne, "The National Advanced Driving Simulator: Potential applications to ITS and AHS research," in Proceeding of the 6th Annual meeting of the Intelligent Transportation Society, Washington D.C, USA, 1996, pp. 700-710.

[11] M. A. Djeziri, R. Merzouki, and B. O. Bouamama, "Robust monitoring of electric vehicle with structured and unstructured uncertainties," IEEE Transaction on Vehicular Technology, vol. 58, no. 9, pp. 4710-4719, 2009.

[12] H. Mohellebi, S. Espié, H. Arioui, A. Amouri, and A. Kheddar, "Low cost motion platform for driving simulator," in 5th International Conference on Machine Automation (ICMA04), Osaka, Japan, Nov. 2004.

[13] L. Nehaoua, H. Mohellebi, A. Amouri, H. Arioui, and A. Kheddar, "Design and control of a small-clearance driving simulator," IEEE Transactions on Vehicular Technology, vol. 57, no. 2, pp. 736-746, 2008.

[14] H. Arioui, L. Nehaoua, S. Hima, N. Séguy, and S. Espié, "Design and control of a small-clearance driving simulator," IEEE/ASME Transactions on Mechatronics, vol. 15, no. 5, pp. 805-818, 2010.

[15] P. R. Grant and L. D. Reid, "Motion washout filter tuning: Rules and requirements," Journal Of Aircraft, vol. 34, pp. 145-151, Mar.-Apr. 1997.

[16] M. A. Nahon and L. D. Reid, "Simulator Motion-Drive Algorithms: A Designer's Perspective," Journal of Guidance and Dynamics, vol. 13, pp. 356-362, July 1989 .

[17] M. Idan and M. A. Nahon, "Off-Line Comparison of Classical and Robust Flight Simulator Motion Control," Journal of Guidance and Dynamics, vol. 22, pp. 702-709, Sept.-Oct. 1999.

[18] L. Reid and M. Nahon, "Response of Airline Pilots to Variations in Flight Simulator Motion Algorithms," in Journal of Aircraft, vol. 25, July 1988, pp. 639-646.

[19] J. Neimer, H. Mohellebi, S. Espié, and A. Kheddar, "Optimization of linear motion base dedicated to normal driving conditions," in Driving Simulator Conference (DSC05), Orlando, Florida, 30 nov-2 dec 2005.

[20] S. Marchant, "Réalisation d'un simulateur automobile," in Master Thesis, Ecole Nationale de l'Aviation Civile, France, Aug. 1995.

[21] R. Featherstone, Rigid Body Dynamics Algorithms. Springer, 2008.

[22] J. L. Meiry, "The Vestibular System and Human Dynamic Space Orientation," in M.I.T PhD Thesis, Cambridge, Massachusetts, June 1965.

[23] G. Reymond, "Contribution respective des stimuli visuels, vestibulaires et proprioceptifs dans la perception du mouvement du conducteur," in PhD Thesis, University of Paris6, France, Dec. 2000.

[24] L. Harris, M. Jenkin, D. Zikovitz, and F. Redlick, "Simulating SelfMotion I: Cues for the Perception of Motion," in Springer-Verlag Virtual Reality, vol. 6, Sept. 2002, pp. 75-85.

[25] F. Cardullo, R. Telban, and J. Houck, "Motion cueing algorithms: A human centered approach," in 5th International Symposium on Aeronautical Sciences, Zhukovsky, Russia, 1999.

[26] S. Espie, "Vehicle-driven simulator versus traffic-driven simulator: the inrets approach-driving simulation conference," in Driving Simulation Conference (DSC99), Paris, France, 1999. 